Proyecciones Journal of Mathematics

Vol. 37, N ${ }^{o}$ 2, pp. 317-344, June 2018.

Universidad Católica del Norte

Antofagasta - Chile

\title{
Solutions and stability of a variant of Wilson's functional equation
}

\author{
Elqorachi Elhoucien \\ Ibn Zohr University, Morocco \\ and \\ Redouani Ahmed \\ Ibn Zohr University, Morocco \\ Received : May 2017. Accepted : April 2018
}

\begin{abstract}
In this paper we will investigate the complex-valued solutions and stability of the generalized variant of Wilson's functional equation

$$
(E): \quad f(x y)+\chi(y) f(\sigma(y) x)=2 f(x) g(y), x, y \in G,
$$

where $G$ is a group, $\sigma$ is an involutive morphism of $G$ and $\chi$ is a character of $G$. (a) We solve $(E)$ when $\sigma$ is an involutive automorphism, and we obtain some properties about solutions of $(E)$ when $\sigma$ is an involutive anti-automorphism. (b) We obtain the Hyers Ulam stability of equation $(E)$. As an application, we prove the superstability of the functional equation $f(x y)+\chi(y) f(\sigma(y) x)=2 f(x) f(y), x, y \in G$.
\end{abstract}

Keywords Group, Semigroup-Involution, D'Alembert's equation, Wilson's equation, Automorphism, Homomorphism, Multiplicative function, Hyers-Ulam stability, Superstability.

Subjclass : Primary 39B82; Secondary 39B32, 39B52. 


\section{Introduction}

D'Alembert's functional equation

$$
f(x+y)+f(x-y)=2 f(x) f(y), x, y \in \mathbf{R}
$$

also called the cosine functional equation has a long history going back to J. d'Alembert. Equation (1.1) plays an important role in determining the sum of two vectors in various Euclidean and non-Euclidean geometries.

The continuous solutions $f: \mathbf{R} \longrightarrow \mathbf{C}$ of d'Alembert's functional equation (1.1) are known: Apart from the trivial solution $f=0$, they are $f_{\lambda}(x)=\cos (\lambda x), x \in \mathbf{R}$ where the parameter $\lambda$ ranges over $\mathbf{C}$ (see for example [1])

Several authors have determined the general solution on groups $f$ : $G \longrightarrow \mathbf{C}$ of the following generalization of d'Alembert's functional equation

$$
f(x y)+f(x \sigma(y))=2 f(x) f(y), x, y \in G
$$

in abelian case and in non abelian case, and where $\sigma$ is an involution of $G$.

Probably the first result for non abelian groups was obtained by Kannappan [20]. Under the condition $f(x y z)=f(y x z)$ for all $x, y, z \in G$, the solutions of equation (1.2) with $\sigma(x)=x^{-1}$, for all $x \in G$ are of the form $f(x)=\frac{\phi(x)+\phi\left(x^{-1}\right)}{2}$, where $\phi$ is multiplicative.

There has been quite a development of the theory of d'Alembert's functional equation (1.1) during the last years, on non abelian groups, as shown in works by Dilian Yang about compact groups [9, 10, 11], Stetkær [29] for step 2 nilpotent groups, Friis [15] for results on Lie groups and Davison [7, 8] for general groups, even monoids. The most comprehensive recent study is by Stetkær [28].

Recently, Stetkær [26] obtained the complex valued solutions of the following version of d'Alembert's functional equation

$$
f(x y)+\chi(y) f\left(x y^{-1}\right)=2 f(x) f(y), x, y \in G,
$$

where $\chi: G \longrightarrow \mathbf{C}$ is a character of $G$. The non-zero solutions of equation (1.3) are the normalized traces of certain representation of the group $G$ on 
$\mathbf{C}^{2}$.

The year after Stetkær [25] obtained the complex valued solutions of the following variant of d'Alembert's functional equation

$$
f(x y)+f(\sigma(y) x))=2 f(x) f(y), x, y \in G,
$$

where $\sigma$ is an involutive homomorphism of $G$. The solutions of equation (1.4) are of the form $f(x)=\frac{\varphi(x)+\varphi(\sigma(x))}{2}, x \in G$, where $\varphi$ is multiplicative.

We refer also to Wilson's first generalization of d'Alembert's functional equation:

$$
f(x+y)+f(x-y)=2 f(x) g(y), x, y \in \mathbf{R} .
$$

For more about the functional equation (1.5) see Aczél ([1], Section 3.2.1 and 3.2.2). The solution formulas of equation (1.5) are known.

In [12] Ebanks and Stetkær studied the solutions $f, g: G \longrightarrow \mathbf{C}$ of Wilson's functional equation

$$
f(x y)+f\left(x y^{-1}\right)=2 f(x) g(y), x, y \in G
$$

and the following variant of Wilson's functional equation (see also [31])

$$
f(x y)+f\left(y^{-1} x\right)=2 f(x) g(y), x, y \in G .
$$

They solved (1.7) and they obtained some new results about (1.6).

The stability of d'Alembert's functional equation was first obtained by Baker [4] when the following theorem was proved.

Theorem 1.1 (4). (Superstability of d'Alembert's functional equation) Let $G$ be an abelian group. If a function $f: G \longrightarrow \mathbf{C}$ satisfies the inequality

$$
|f(x+y)+f(x-y)-2 f(x) f(y)| \leq \delta
$$

for some $\delta>0$ and for all $x, y \in G$, then either $f$ is bounded on $G$ or $f(x+y)+f(x-y)=2 f(x) f(y)$ for all $x, y \in G$.

A different generalization of Baker's result was given by L. Székelyhidi $[33,34]$. It involves an interesting generalization of the class of bounded 
function on a group or semigroup. For other stability and superstability results, we can see for example [2], [3], [6], [13], [16], [17], [23] and [5] for general groups.

Various stability results of Wilson's functional equation and its generalization are obtained. The number of papers in this subject is very high, hence, it is not realistic to try to refer to all. The interested reader should refer to [14], [18] and [19] for a thorough account on the subject of stability of functional equations.

The main purpose of this paper is to study the solutions and stability of the more general variant of Wilson's functional equation

$$
f(x y)+\chi(y) f(\sigma(y) x)=2 f(x) g(y), x, y \in G,
$$

where $G$ is a group, $\chi$ is a character of $G$ such that $\chi(x \sigma(x))=1$, for all $x \in G, \sigma$ is an involutive morphism of $G$. That is, $\sigma(x y)=\sigma(y) \sigma(x)$ and $\sigma(\sigma(x))=x$ for all $x, y \in G$ or $\sigma(x y)=\sigma(x) \sigma(y)$ and $\sigma(\sigma(x))=x$ for all $x, y \in G$.

On abelian groups the solutions and stability of the functional equation (1.8) are known, so the contributions of the present papers to theory of trigonometric functional equation and their stability in the non-abelian case.

We solve (1.8) when $\sigma$ is an involutive automorphism, and we obtain some properties of the solutions of equation (1.8) when $\sigma$ is an involutive anti-automorphism. The solutions are expressed in terms of characters and additive functions. Furthermore, we obtain the Hyers Ulam stability of equation (1.8). As an application we prove the superstability of the functional equation

$$
f(x y)+\chi(y) f(\sigma(y) x)=2 f(x) f(y), x, y \in G .
$$

Our results are natural extensions of the previous papers mentioned above. In particular the structure and the form of the solutions follow the same pattern as in the previous papers. 


\section{Stability of the functional equation (1.8), where $\sigma$ is an involutive anti-automorphism of $G$.}

In this section $\sigma$ is an involutive anti-automorphism of $G$, that is $\sigma(x y)=$ $\sigma(y) \sigma(x)$ and $\sigma(\sigma(x))=x$, for all $x, y \in G$. The following theorem is one of the main results of the present paper. Recalling that $m_{h}$ for any complexvalued function $h$ on $G$ is defined by $m_{h}(x)=2 h(x)^{2}-h\left(x^{2}\right), x \in G$. We have

Theorem 2.1. Let $\delta \geq 0$. Let $\sigma$ be an involutive anti-automorphism of $G$. Let $\chi$ be a unitary character of $G$ such that $\chi(x \sigma(x))=1$ for all $x \in G$.

Suppose that the functions $f, g: G \longrightarrow \mathbf{C}$ satisfy the inequality

$$
|f(x y)+\chi(y) f(\sigma(y) x)-2 f(x) g(y)| \leq \delta
$$

for all $x, y \in G$. Under these assumptions the following statements hold:

(1) If $f$ is unbounded, then

(i) $g$ is central. That is $g(x y)=g(y x)$, for all $x, y \in G ; m_{g}: G \longrightarrow \mathbf{C}^{*}$ is multiplicative.

(ii) If $\sigma(x)=x^{-1}$, then $g(x)=\chi(x) g(\sigma(x))$ for all $x \in G$ and $\check{\chi} m_{g}(G) \subseteq\{\mp 1\}$, where $\check{\chi}(x)=\chi\left(x^{-1}\right), x \in G$.

(iii) $g(\mathrm{x})=\mathrm{m}_{g}(x) g\left(x^{-1}\right)$ for all $x \in G$ and

$$
g(x y)+m_{g}(y) g\left(x y^{-1}\right)=2 g(x) g(y) \text { for all } x, y \in G \text {. }
$$

(2) If $g$ is unbounded and $f \neq 0$, then

(v) the pair $(f, g)$ satisfies the functional equation (1.8). Furthermore,

(vi) $m_{g}: G \longrightarrow \mathbf{C}^{*}$ is multiplicative. If $\sigma(x)=x^{-1}$ then $g(x)=\chi(x) g(\sigma(x))$ for all $x \in G$ and $\check{\chi} m_{g}(G) \subseteq\{\mp 1\}$.

(vii) $g(x)=m_{g}(x) g\left(x^{-1}\right)$, $\chi(y) f(\sigma(y) x y)=m_{g}(y) f(x)$ for all $\mathrm{x}, \mathrm{y} \in G$.

(viii) The pair $(f, g)$ satisfies the functional equation

$$
f(x y)+m_{g}(y) f\left(x y^{-1}\right)=2 f(x) g(y), x, y \in G
$$

and $g$ satisfies equation ((2.2)). 
Proof. All technical methods that are needed in our discussion are due to Stetkær [21]. (1) We let $L$ and $R$ denote respectively: the left and right regular representation of $G$ with respect to $\sigma$ on functions on $G$. That is, $[L(y) h](x)=h(\sigma(y) x)$ and $R(y) h(x)=h(x y)$ for $x, y \in G$ and $h: G \longrightarrow \mathbf{C}$. We notice here that $L(x) R(y)=R(y) L(x)$ and $L(y z) h=L(y)[L(z) h]$, $R(y z) h=R(y)[R(z) h]$ for all $x, y \in G$ and for all function $h: G \longrightarrow \mathbf{C}$.

Thus, inequality (2.1) can be written as follows

$$
\|[R(y)+\chi(y) L(y)] f-2 g(y) f\|_{\infty} \leq \delta
$$

for all $y \in G$ where for any bounded complex-valued function $h$ on $G$ the norm $\|h\|_{\infty}$ is defined by $\|h\|_{\infty}=\sup _{x \in G}|h(x)|$. Applying the operator $R(z)+\chi(z) L(z)$ to the bounded function $R(y) f+\chi(y) L(y) f-2 g(y) f$ we get after reduction that

$$
\|(R(z)+\chi(z) L(z))[R(y)+\chi(y) L(y)] f-2 g(y)(R(z) f+\chi(z) L(z)) f\|_{\infty}
$$

$$
\begin{gathered}
=\|[(R(z y)+\chi(z y) L(z y)) f-2 g(z y) f+2 g(z y) f+[\chi(y) R(z) L(z)+\chi(z) L(z) R(y)] f \\
-2 g(y)(R(z) f+\chi(z) L(z) f-2 g(z) f)-4 g(z) g(y) f \|_{\infty} .
\end{gathered}
$$

By using (2.4) and that $\|(R(z)+\chi(z) L(z)) h\|_{\infty} \leq 2\|h\|_{\infty}$ for all complex bounded functions $h$ on $G$ we obtain

$\|2 g(z y) f+[\chi(y) R(z) L(y)+\chi(z) L(z) R(y)] f-4 g(z) g(y) f\|_{\infty} \leq 3 \delta+2|g(y)| \delta$

for all $z, y \in G$.

(1) (i) Interchanging $z$ and $y$ in (2.6) and substracting the result obtained from (2.6) we get

$$
\|[g(z y)-g(y z)] f\|_{\infty} \leq|g(z)| \delta+|g(y)| \delta+3 \delta .
$$

Since $f$ is assumed to be unbounded, then $g$ is central.

Setting $y=z$ in $(2.6)$, we obtain 


$$
\left\|\left(2 g(y)^{2}-g\left(y^{2}\right)\right) f-\chi(y) R(y) L(y) f\right\|_{\infty} \leq|g(y)| \delta+\frac{3}{2} \delta .
$$

That is,

$$
\left|\left(2 g(y)^{2}-g\left(y^{2}\right)\right) f(x)-\chi(y) f(\sigma(y) x y)\right| \leq|g(y)| \delta+\frac{3}{2} \delta
$$

for all $x, y \in G$. Which can be written as follows.

$$
\left\|m_{g}(y) f-\chi(y) \mu(y) f\right\|_{\infty} \leq|g(y)| \delta+\frac{3}{2} \delta,
$$

where $[\mu(y) h](x)=h(\sigma(y) x y)$. Noting that $\mu(y z)=\mu(y) \mu(z)$ for all $z, y \in$ $G$. By using inequality (2.8) we have

$$
\begin{gathered}
\left|m_{g}(y z) f(x)-\chi(y) \chi(z) f(\sigma(z) \sigma(y) x y z)\right| \leq|g(y z)| \delta+\frac{3}{2} \delta \\
\left|m_{g}(y) f(x)-\chi(y) f(\sigma(y) x y)\right| \leq|g(y)| \delta+\frac{3}{2} \delta
\end{gathered}
$$

and

$$
\left|m_{g}(z) f(\sigma(y) x y)-\chi(z) f(\sigma(z) \sigma(y) x y z)\right| \leq|g(z)| \delta+\frac{3}{2} \delta .
$$

So, by using the triangle inequality we get

$$
\begin{gathered}
\left|m_{g}(y z) f(x)-m_{g}(y) m_{g}(z) f(x)\right| \leq\left|m_{g}(y z) f(x)-\chi(y) \chi(z) f(\sigma(z) \sigma(y) x y z)\right| \\
+\left|\chi(y) \chi(z) f(\sigma(z) \sigma(y) x y z)-m_{g}(y) m_{g}(z) f(x)\right| \\
\leq\left|m_{g}(y z) f(x)-\chi(y) \chi(z) f(\sigma(z) \sigma(y) x y z)\right| \\
+\left|\chi(y) \chi(z) f(\sigma(z) \sigma(y) x y z)-m_{g}(z) \chi(y) f(\sigma(y) x y)\right| \\
+\left|m_{g}(z) \chi(y) f(\sigma(y) x y)-m_{g}(y) m_{g}(z) f(x)\right| \\
\leq|g(y z)| \delta+\frac{3}{2} \delta+\left|m_{g}(z)\right|\left|\chi(y) f(\sigma(y) x y)-m_{g}(y) f(x)\right| \\
+|\chi(y)|\left|\chi(z) f(\sigma(z) \sigma(y) x y z)-m_{g}(z) f(\sigma(y) x y)\right| \\
\leq|g(y z)| \delta+\frac{3}{2} \delta+\left|m_{g}(z)\right|\left[|g(y)| \delta+\frac{3}{2} \delta\right]+|\chi(y)|\left[|g(z)| \delta+\frac{3}{2} \delta\right] .
\end{gathered}
$$

From the assumption that $f$ is unbounded we get $m_{g}(y z)=m_{g}(y) m_{g}(z)$ for all $y, z \in G$. On the other hand if $m_{g}=0$, then if we put $y=e$ in (2.8) we obtain $f$ bounded. But $f$ is unbounded, so $m_{g}(G) \subseteq \mathbf{C}^{*}$. 
(ii) Now, let $a \in G$ be arbitrary. First case: Assume that $g$ is a bounded function on $G$. Setting $x=e$ in (2.1), we obtain

$$
|f(y)+\chi(y) f(\sigma(y))-2 f(e) g(y)| \leq \delta
$$

for all $y \in G$. Which implies that

$$
|f(y)+\chi(y) f(\sigma(y))| \leq \delta+2|f(e)| M
$$

for all $y \in G$ and where $M=\sup _{x \in G}|g(x)|$. Thus, from $\chi(a \sigma(a))=1$, $2 f(x)[g(a)-\chi(a) g(\sigma(a))]$ can be written as follows

$$
\begin{aligned}
\mid 2 f(x)[ & g(a)-\chi(a) g(\sigma(a))]=2 f(x) g(a)-f(x a)-\chi(a) f(\sigma(a) x) \\
& +\chi(a)[f(x \sigma(a))+\chi(\sigma(a)) f(a x)-2 f(x) g(\sigma(a))] \\
& +f(x a)+\chi(a) f(\sigma(a) x)-\chi(a) f(x \sigma(a))-f(a x) .
\end{aligned}
$$

Since

$$
\begin{gathered}
f(x a)+\chi(a) f(\sigma(a) x)-\chi(a) f(x \sigma(a))-f(a x) \\
=f(x a)+\chi(a) f(\sigma(a) x)-\chi(a)[f(x \sigma(a))+\chi(x \sigma(a)) f(a \sigma(x))]+\chi(x) f(a \sigma(x)) \\
-[f(a x)+\chi(a x) f(\sigma(x) \sigma(a))]+\chi(a x) f(\sigma(x) \sigma(a)) \\
=\chi(x)[f(a \sigma(x))+\chi(\sigma(x)) f(\sigma(\sigma(x)) a)-2 f(a) g(\sigma(x))]+2 \chi(x) f(a) g(\sigma(x)) \\
+\chi(a)[f(\sigma(a) x)+\chi(x) f(\sigma(x) \sigma(a))-2 f(\sigma(a)) g(x)]+2 \chi(a) f(\sigma(a)) g(x) \\
-\chi(a)[f(x \sigma(a))+\chi(x \sigma(a)) f(a \sigma(x))]-[f(a x)+\chi(a x) f(\sigma(x) \sigma(a))] .
\end{gathered}
$$

From inequalities (2.1), (2.10) and $|\chi(x)|=1$ we get

$$
|2 f(x)[g(a)-\chi(a) g(\sigma(a))]| \leq 4 \delta+2|f(\sigma(a))| M+2|f(a)| M+4|f(e)| M .
$$

for all $x \in G$. By using the unboundedness of $f$ we get $g(a)=\chi(a) g(\sigma(a))$.

Second case: If $g$ is unbounded. For all $x, y, z \in G$ we have

$$
\begin{gathered}
|2 g(z)||f(x y)+\chi(y) f(\sigma(y) x)-2 f(x) g(y)| \\
=|2 f(x y) g(z)+2 \chi(y) f(\sigma(y) x) g(z)-4 f(x) g(y) g(z)|
\end{gathered}
$$




$$
\begin{gathered}
\leq|-f(x y z)-\chi(z) f(\sigma(z) x y)+2 f(x y) g(z)| \\
+|\chi(y)[-f(\sigma(y) x z)-\chi(z) f(\sigma(z) \sigma(y) x)+2 f(\sigma(y) x) g(z)]| \\
+|+f(x y z)+\chi(y z) f(\sigma(z) \sigma(y) x)-2 f(x) g(y z)| \\
+|-f(x z y)-\chi(z y) f(\sigma(y) \sigma(z) x)+2 f(x) g(z y)| \\
+|\chi(z) f(\sigma(z) x y)+\chi(z y) f(\sigma(y) \sigma(z) x)-2 \chi(z) f(\sigma(z) x) g(y)| \\
+|f(x z y)+\chi(y) f(\sigma(y) x z)-2 f(x z) g(y)| \\
+2|f(x)||g(y z)-g(z y)|+2 g(y)|f(x z)+\chi(z) f(\sigma(z) x)-2 f(x) g(z)| . \\
\leq \delta+\delta+\delta+\delta+|\chi(z)| \delta+0 \times 2|f(x)| \delta+2|g(y)| \delta=6 \delta+2|g(y)| \delta .
\end{gathered}
$$

Using that $g$ is unbounded, we get that the pair $(f, g)$ satisfies the functional equation (1.8). For the rest of the proof of (ii) we assume that $\sigma(x)=x^{-1}$ for all $x \in G$. We discuss two subcases: First subcase: Assume either $f(a) \neq 0$ or $f(e) \neq 0$. The pair $(f, g)$ satisfies equation (1.8) with $\sigma(x)=x^{-1}$ on the abelian subgroup $\langle a\rangle$ generated by $a$, then on the abelian subgroup $\langle a\rangle$ we have $f\left(x y^{-1}\right)+\chi\left(y^{-1}\right) f(x y)=2 f(x) g(\sigma(y))$, since $\chi\left(y y^{-1}\right)=1$ hence we get $\chi(y) f\left(x y^{-1}\right)+f(x y)=2 f(x) \chi(y) g\left(y^{-1}\right)=$ $2 f(x) g(y), x, y \in G$. Then we have $g(y)=\chi(y) g(\sigma(y))$ for all $y \in\langle a\rangle$. In particular $g(a)=\chi(a) g(\sigma(a))$.

Second subcase: Assume that $f(a)=0$ and $f(e)=0$. Setting $x=e$ in (1.8) with $\sigma(x)=x^{-1}$, we obtain

$$
f(y)+\chi(y) f\left(y^{-1}\right)=0
$$

for all $y \in G$. In particular $f\left(a^{-1}\right)=0$. By using the above computation we get

$$
\begin{aligned}
\mid 2 f(x)[ & \left.g(a)-\chi(a) g\left(a^{-1}\right)\right]=2 f(x) g(a)-f(x a)-\chi(a) f\left(a^{-1} x\right) \\
& +\chi(a)\left[f\left(x a^{-1}\right)+\chi\left(a^{-1}\right) f(a x)-2 f(x) g\left(a^{-1}\right)\right] \\
+ & f(x a)+\chi(a) f\left(a^{-1} x\right)-\chi(a) f\left(x a^{-1}\right)-f(a x) .
\end{aligned}
$$

Since

$$
\begin{gathered}
f(x a)+\chi(a) f\left(a^{-1} x\right)-\chi(a) f\left(x a^{-1}\right)-f(a x) \\
=f(x a)+\chi(a) f\left(a^{-1} x\right)-\chi(a)\left[f\left(x a^{-1}\right)+\chi\left(x a^{-1}\right) f(a \sigma(x))\right]+\chi(x) f(a \sigma(x)) \\
-\left[f(a x)+\chi(a x) f\left(\sigma(x) a^{-1}\right)\right]+\chi(a x) f\left(\sigma(x) a^{-1}\right)
\end{gathered}
$$




$$
\begin{gathered}
=\chi(x)[f(a \sigma(x))+\chi(\sigma(x)) f(\sigma(\sigma(x)) a)-2 f(a) g(\sigma(x))]+2 \chi(x) f(a) g(\sigma(x)) \\
+\chi(a)\left[f\left(a^{-1} x\right)+\chi(x) f\left(\sigma(x) a^{-1}\right)-2 f\left(a^{-1}\right) g(x)\right]+2 \chi(a) f\left(a^{-1}\right) g(x) \\
-\chi(a)\left[f\left(x a^{-1}\right)+\chi\left(x a^{-1}\right) f(a \sigma(x))\right]-\left[f(a x)+\chi(a x) f\left(\sigma(x) a^{-1}\right)\right] .
\end{gathered}
$$

So, we get $2 f(x)\left[g(a)-\chi(a) g\left(a^{-1}\right)\right]=0$, which implies that $g(a)=$ $\chi(a) g\left(a^{-1}\right)$.

On the other hand we have $m_{g}\left(y^{-1}\right)=2 g\left(y^{-1}\right)^{2}-g\left(\left(y^{-1}\right)^{2}\right)$ $=2 \chi\left(y^{-1}\right)^{2} g(y)^{2}-\chi\left(y^{-1}\right)^{2} g\left(y^{2}\right)=\chi\left(y^{-1}\right)^{2} m_{g}(y)$.

So, we get $m_{g}(y) m_{g}\left(y^{-1}\right)=m_{g}(e)=1=\left(\chi\left(y^{-1}\right) m_{g}(y)\right)^{2}$. Which implies that $\check{\chi} m_{g}(G) \subseteq\{\mp 1\}$.

(iii) The formula $2 f(x)\left[g(y)-m_{g}(y)\left(y^{-1}\right)\right]$ can be written as follows

$$
\begin{gathered}
2 f(x)\left[g(y)-m_{g}(y) g\left(y^{-1}\right)\right]= \\
{[-f(x y)-\chi(y) f(\sigma(y) x)+2 f(x) g(y)]} \\
\left.+m_{g}(y)\left[f\left(x y^{-1}\right)+\chi\left(y^{-1}\right) f\left(\sigma\left(y^{-1}\right) x\right)\right)-2 f(x) g\left(y^{-1}\right)\right] \\
+f(x y)-\chi\left(y^{-1}\right) m_{g}(y) f\left(\sigma\left(y^{-1}\right) x\right)+\chi(y) f(\sigma(y) x)-m_{g}(y) f\left(x y^{-1}\right) .
\end{gathered}
$$

Once again we have

$$
\begin{gathered}
f(x y)-\chi\left(y^{-1}\right) m_{g}(y) f\left(\sigma\left(y^{-1}\right) x\right)+\chi(y) f(\sigma(y) x)-m_{g}(y) f\left(x y^{-1}\right) \\
=f\left(\sigma(y) \sigma\left(y^{-1}\right) x y\right)-\chi\left(y^{-1}\right) m_{g}(y) f\left(\sigma\left(y^{-1}\right) x\right)+\chi(y) f\left(\sigma(y) x y^{-1} y\right) \\
-m_{g}(y) f\left(x y^{-1}\right) \\
=[\mu(y) f]\left(\sigma\left(y^{-1}\right) x\right)-\chi\left(y^{-1}\right) m_{g}(y) f\left(\sigma\left(y^{-1}\right) x\right)+\chi(y)[\mu(y) f]\left(x y^{-1}\right) \\
-m_{g}(y) f\left(x y^{-1}\right)
\end{gathered}
$$

and from inequality (2.8) and the triangle inequality we obtain

$$
\begin{aligned}
& \left.\mid[\mu(y) f]\left(\sigma\left(y^{-1}\right) x\right)-\chi\left(y^{-1}\right) m_{g}(y) f\left(\sigma\left(y^{-1}\right) x\right)\right)+\chi(y)[\mu(y) f]\left(x y^{-1}\right) \\
& -m_{g}(y) f\left(x y^{-1}\right) \mid \\
& \leq 2|g(y)| \delta+3 \delta
\end{aligned}
$$

Now, from inequalities (2.1) and (2.13) we get 


$$
\left|2 f(x)\left[g(y)-m_{g}(y) g\left(y^{-1}\right)\right]\right| \leq\left|m_{g}(y)\right| \delta+2|g(y)| \delta+4 \delta
$$

for all $x, y \in G$. Since $f$ is unbounded then we have $g(y)=m_{g}(y) g\left(y^{-1}\right)$ for all $y \in G$.

(vi) Let us consider

$$
2 f(x)\left[g(z y)+m_{g}(y) g\left(z y^{-1}\right)-2 g(y) g(z)\right]
$$

$$
\begin{aligned}
=[ & 2 g(z y) f(x)-4 g(y) g(z) f(x)+\chi(y)(R(z) L(y) f)(x)+\chi(z)(L(z) R(y) f)(x)] \\
& +2 f(x) m_{g}(y) g\left(z y^{-1}\right)-\chi(y)(R(z) L(y) f)(x)-\chi(z)(L(z) R(y) f)(x) .
\end{aligned}
$$

Since

$$
\begin{gathered}
2 f(x) m_{g}(y) g\left(z y^{-1}\right)-\chi(y)(R(z) L(y) f)(x)-\chi(z)(L(z) R(y) f)(x) \\
=2 f(x) m_{g}(y) g\left(z y^{-1}\right)-\chi(y) f\left(\sigma(y) x z y^{-1} y\right)-\chi(z) f\left(\sigma(y) \sigma\left(y^{-1}\right) \sigma(z) x y\right) \\
=2 f(x) m_{g}(y) g\left(z y^{-1}\right)-\chi(y) f\left(\sigma(y) x z y^{-1} y\right)+m_{g}(y) f\left(x z y^{-1}\right)-m_{g}(y) f\left(x z y^{-1}\right) \\
\left.-\chi(z) f\left(\sigma(y) \sigma\left(y^{-1}\right) \sigma(z) x y\right)+\chi\left(y^{-1}\right) \chi(z) m_{g}(y) f\left(\sigma\left(y^{-1}\right) \sigma(z) x\right)\right) \\
\left.-\chi\left(y^{-1}\right) \chi(z) m_{g}(y) f\left(\sigma\left(y^{-1}\right) \sigma(z) x\right)\right) \\
=-\chi(y)[\mu(y) f]\left(x z y^{-1}\right)+m_{g}(y) f\left(x z y^{-1}\right) \\
\left.-\chi(z)[\mu(y) f]\left(\sigma\left(y^{-1}\right) \sigma(z) x\right)+\chi\left(y^{-1}\right) \chi(z) m_{g}(y) f\left(\sigma\left(y^{-1}\right) \sigma(z) x\right)\right) \\
-m_{g}(y)\left[f\left(x z y^{-1}\right)+\chi\left(z y^{-1}\right) f\left(\sigma\left(y^{-1}\right) \sigma(z) x\right)-2 f(x) g\left(z y^{-1}\right)\right] .
\end{gathered}
$$

From inequalities (2.1), (2.6) and $|\chi(z)|=1$ we obtain

$$
\left|2 f(x)\left[g(z y)+m_{g}(y) g\left(z y^{-1}\right)-2 g(y) g(z)\right]\right| \leq 6 \delta+4|g(y)| \delta+\left|m_{g}(y)\right| \delta
$$

for all $x, y, z \in G$. Since $f$ is unbounded, then $g$ satisfies the functional equation $((2.2))$

(2) We assume that $g$ is unbounded and $f \neq 0$. By simple computations we get that $f$ is unbounded. Now, by using some computations used in (ii) we get that the pair $(f, g)$ satisfies the functional equation (1.8).

Now, by using (2.8) with $\delta=0$ we get 


$$
m_{g}(y) f(x)=\chi(y) f(\sigma(y) x y)
$$

for all $x, y \in G$.

So if we replace $x$ by $x y^{-1}$ in $(2.16)$ we obtain $m_{g}(y) f\left(x y^{-1}\right)=\chi(y) f(\sigma(y) x)$ and equation (1.8) can be written as follows $f(x y)+m_{g}(y) f\left(x y^{-1}\right)=$ $2 f(x) g(y)$ for all $x, y \in G$. For the proof of other properties we use case (1) with $\delta=0$. This completes the proof of theorem.

Corollary 2.2. Let $\delta \geq 0$. Let $\sigma$ be an involutive anti-automorphism of $G$.

Let $\chi$ be a unitary character of $G$ such that $\chi(x \sigma(x))=1$ for all $x \in G$. Suppose that the function $f: G \longrightarrow \mathbf{C}$ satisfies the inequality

$$
|f(x y)+\chi(y) f(\sigma(y) x)-2 f(x) f(y)| \leq \delta
$$

for all $x, y \in G$. Then, either $f$ is bounded or $f$ satisfies equation (1.9).

As an application we get some properties of the solutions of equation (1.8), where $\sigma$ is an involutive anti-automorphism. By using the above Theorem for $\delta=0,[12]$, Proposition 2, Theorem 3 and Corollary 6], [[39], Theorem 6.1, Theorem 10.1 and Corollary 10.2] we obtain the following theorem.

For later use, we recall (see for example [12] that a function $f: G \longrightarrow \mathbf{C}$ is said to be abelian, if $f\left(x_{\sigma(1)} x_{\sigma(2)} \ldots x_{\sigma(n)}\right)=f\left(x_{1} x_{2} \ldots x_{n}\right)$ for all $x_{1}, x_{2}, \ldots, x_{n} \in G$, all permutations $\sigma$ and all $n=1,2, \ldots$

Theorem 2.3. Let $\sigma$ be an involutive anti-automorphism of $G$. Let $\chi$ be a unitary character of $G$ such that $\chi(x \sigma(x))=1$ for all $x \in G$. Let the pair $f, g: G \longrightarrow \mathbf{C}$ be a solution of the variant of Wilson's functional equation (1.8) such that $f \neq 0$.

(1) If $f$ is a nonzero central function. Then,

(i) $f=f(e) g$, when $g$ is non abelian.

(ii) When $g$ is abelian $g$ has the form $g=\frac{\psi+\chi \psi \circ \sigma}{2}$ where $\psi: G \longrightarrow \mathbf{C}^{*}$ is a character. If $\psi \neq \chi \psi \circ \sigma$, then $f=\alpha(\psi-\chi \psi \circ \sigma) / 2+\beta(\psi+\chi \omega \circ \sigma) / 2$ for some $\alpha, \beta \in \mathbf{C}$. If $\psi=\chi \psi \circ \sigma$, then $f=\psi a+\beta \psi$ for some additive map $a$ : $G \longrightarrow \mathbf{C}$ and some $\beta \in \mathbf{C}$.

(2) (i) $g(e)=1, g$ is central, and $g=\chi g \circ \sigma, g=m_{g} \check{g}$.

(ii) $m_{g}: G \longrightarrow \mathbf{C}^{*}$ is multiplicative. 
(iii) $\chi(y) f(\sigma(y) x y)=m_{g}(y) f(x), \chi(y) f(\sigma(y) x)=m_{g}(y) f\left(x y^{-1}\right)$ for all $x, y \in G$.

(iv) $g(x y)+m_{g}(y) g\left(x y^{-1}\right)=2 g(x) g(y)$ for all $x, y \in G$.

(v) $f(x y)+m_{g}(y) f\left(x y^{-1}\right)=2 f(x) g(y)$ for all $x, y \in G$.

(vi) $f=-\chi f \circ \sigma$ if and only if $f(e)=0$

(vii) The even part and the odd part of $f: f_{e}(x)=\frac{f(x)+\chi(x) f(\sigma(x))}{2}, f_{o}(x)=$ $\frac{f(x)-\chi(x) f(\sigma(x))}{2}$ and $\chi f \circ \sigma$ satisfy (1.8) with $g$ unchanged.

(ix) $f_{e}=f(e) g$. In particular $f_{e}=0$ ( $f$ is odd) if and only if $f(e)=0$

(x) The odd part $f_{o}$ of $f$ satisfies

$$
f_{o}(x y)+f_{o}(y x)=2 f_{o}(x) g(y)+2 f_{o}(y) g(x)
$$

for all $x, y \in G$..

(3) For the rest we assume that $\sigma(x)=x^{-1}$ for all $x \in G$.

(i) $\check{\chi} m_{g}(G) \subseteq\{\mp 1\}$

If $m_{g}=\chi$, then either

(i) $g$ is non-abelian and $f=f(e) g$, or

(iii) $f$ and $g$ are both abelian, in which case (1) applies.

(4) If $m_{g} \neq \chi$, then $f=-\chi f \circ \sigma$ ( $f$ is odd).

\section{Solutions and Stability of the functional equation (1.8), where $\sigma$ is an involutive homomorphism of $G$}

In this section $\sigma$ is an involutive homomorphism of $G$, that is $\sigma(x y)=$ $\sigma(x) \sigma(y)$ and $\sigma(\sigma(x))=x$, for all $x, y \in G$. In the following theorem, we obtain the solutions of the functional equation (1.8) on groups. It turns out that, like on abelian groups, only multiplicative and additive functions occur in the solution formulas.

Theorem 3.1. Let $G$ be a groups, $\sigma: G \longrightarrow G$ a multiplicative function such that $\sigma \circ \sigma=I$, where $I$ denotes the identity map, and $\chi: G \longrightarrow \mathbf{C}$ be a multiplicative function on $G$ such that $\chi(x \sigma(x))=1$ for all $x \in G$.

The solutions $f, g$ of the functional equation (1.8) are the following pairs of functions, where $m: G \longrightarrow \mathbf{C}$ denotes an homomorphism and $\alpha, c \in \mathbf{C}^{*}$. 
(i) $f=0$ and $g$ arbitrary.

(ii) $g=\frac{m+\chi m \circ \sigma}{2}$ and $f=\alpha g$.

(iii) $g=\frac{m+\chi m \circ \sigma}{2}$ and $f=\left(c+\frac{\alpha}{2}\right) m-\left(c-\frac{\alpha}{2}\right) m \circ \sigma$ with $(\chi-1) m=(\chi-1) m \circ \sigma$. (iv) $g=m$ and $f=(a+\alpha) m$, where $m=\chi m \circ \sigma$ and $a: G \longrightarrow \mathbf{C}$ is an additive map which satisfies $m(a \circ \sigma+a)=0$.

Proof. It is elementary to check that the cases stated in the Theorem define solutions, so it is left to show that any solution $f, g: G \longrightarrow \mathbf{C}$ of (1.8) falls into one of these cases. We use in the proof similar Stetkær's computations [25]. Let $x, y, z \in G$. If we replace $x$ by $x y$ and $y$ by $z$ in (1.8) we get

$$
f(x y z)+\chi(z) f(\sigma(z) x y)=2 f(x y) g(z) .
$$

On the other hand if we replace $x$ by $\sigma(z) x$ in (1.8), we obtain

$$
\begin{gathered}
f(\sigma(z) x y)+\chi(y) f(\sigma(y) \sigma(z) x)=2 f(\sigma(z) x) g(y) \\
=2 g(y)[\chi(\sigma(z))[2 f(x) g(z)-f(x z)]] .
\end{gathered}
$$

Since,

$$
\begin{aligned}
\chi(y) f(\sigma(y) \sigma(z) x)= & \chi(y) f(\sigma(y z) x)=\chi(y) \chi(\sigma(y z))[2 g(y z) f(x)-f(x y z)] \\
& =\chi(\sigma(z))[2 g(y z) f(x)-f(x y z)],
\end{aligned}
$$

so by using $\chi(z \sigma(z))=1$ we have

$$
\chi(z) f(\sigma(z) x y)+[2 g(y z) f(x)-f(x y z)]=2 g(y)[2 f(x) g(z)-f(x z) .
$$

Subtracting this from (3.1) we get

$$
f(x y z)=g(y z) f(x)+f(x y) g(z)+g(y) f(x z)-2 g(y) f(x) g(z) .
$$

With the notation 


$$
f_{x}(y)=f(x y)-f(x) g(y)
$$

equation (3.3) can be written as follows

$$
f_{a}(x y)=f_{a}(x) g(y)+f_{a}(y) g(x), x, y \in G .
$$

We will in the rest of the proof of Theorem 3.1 need to know the solutions of the functional equation

$$
f(x y)+\chi(y) f(\sigma(y) x)=2 f(x) f(y) ; x, y \in G .
$$

They are obtained in the following lemma.

Lemma 3.2. Let $G$ be a semigroup, $\sigma: G \longrightarrow G$ an homomorphism such that $\sigma \circ \sigma=I$, where $I$ denotes the identity map, and $\chi: G \longrightarrow \mathbf{C}$ be a multiplicative function on $G$ such that $\chi(x \sigma(x))=1$ for all $x \in G$. The solutions $f$ of the functional equation (3.6) are of the form $f=\frac{m+\chi m \circ \sigma}{2}$, where $m: G \longrightarrow \mathbf{C}$ is multiplicative.

Proof. Verifying that $f=\frac{m+\chi m \circ \sigma}{2}$, where $m: G \longrightarrow \mathbf{C}$ is multiplicative, is solution of equation (3.6) consists in simple computations. Let $f$ satisfy the functional equation (3.6), then by using the above computations the pair $f, f_{a}$ satisfies equation

$$
f_{a}(x y)=f_{a}(x) f(y)+f_{a}(y) f(x), x, y \in G .
$$

If $f_{a}=0$ for all $a \in G$ then $f$ is multiplicative. Substituting $f$ into (3.6) we get $\chi(y) f(\sigma(y))=f(y)$ for all $y \in G$. This implies that $f=\frac{\varphi+\chi \varphi \circ \sigma}{2}$, where $f=\varphi$ is multiplicative.

If $f_{a} \neq 0$ for some $a \in G$. From the known solution of the sine addition law (see for example [27], Theorem 4.1]) there exist two multiplicative functions $\chi_{1}, \chi_{2}: G \longrightarrow C$ such that $f=\frac{\chi_{1}+\chi_{2}}{2}$. We can assume that $\chi_{1} \neq \chi_{2}$. Substituting $f=\frac{\chi_{1}+\chi_{2}}{2}$ in (3.6) we get after reduction that

$$
\chi_{1}(x)\left[\chi(y) \chi_{1}(\sigma(y))-\chi_{2}(y)\right]=\chi_{2}(x)\left[\chi_{1}(y)-\chi(y) \chi_{2}(\sigma(y))\right] .
$$

Since $\chi_{1} \neq \chi_{2}$ at least one of $\chi_{1}$ and $\chi_{2}$ is not zero. So, we get $\chi_{1}=\chi_{2} \circ \sigma$, and $f=\frac{\varphi+\chi \varphi \circ \sigma}{2}$, where $\varphi: G \longrightarrow \mathbf{C}$ is multiplicative. This completely describes the solutions of equation (3.6). 
Now, we will find the solutions of equation (1.8). Let $f, g: G \longrightarrow \mathbf{C}$ a solution of equation (1.8). The above computation shows that the pair $f_{a}, g$ satisfies the sine addition formula (3.5) for any $a \in G$. From the known solution of the sine addition formula (see for example [27], Theorem 4.1]) we have the following possibilities.

If $f=0$ we deal with case (i). So during the rest of the proof we will assume that $f \neq 0$. If we replace $a$ by $e$ in (3.4) we get $f_{e}(x)=f(x)-f(e) g(x)$. If $f_{e}=0$, then $f(x)=f(e) g(x)$ for all $x \in G$. Since $f \neq 0$ then $f(e) \neq 0$. Substituting $f=f(e) g$ into (1.8) we find that $g$ satisfies equation (3.6) then there exists $m: G \longrightarrow \mathbf{C}$ multiplicative such that $g=\frac{m+\chi m \circ \sigma}{2}$. We see that we deal with case (ii).

If $f_{e} \neq 0$, the pair $\left(f_{e}, f\right)$ satisfies (3.5) and we known from [27], Theorem 4.1] that there are only the following 3 possibilities:

(1) $f_{e}=\mathrm{cm}$ and $g=m / 2$ for some $m$ multiplicative. Here

$f=f_{e}+f(e) g$. Substituting $f=\left(c+\frac{f(e)}{2}\right) m, g=m / 2$ into (1.8) we find $\left(c+\frac{f(e)}{2}\right) \chi(y) m(x) m(\sigma(y))=0$ for all $x, y \in G$. This case does not apply, because $f \neq 0$.

(2) There exist two different characters $m$ and $M$ on $G$ and a constant $c \in \mathbf{C}^{*}$ such that

$$
g=\frac{m+M}{2} \text { and } f_{e}=c(m-M)
$$

then $f=c(m-M)+f(e) \frac{m+M}{2}=\alpha m-\beta M$, where $\alpha=c+\frac{f(e)}{2}$ and $\beta=c-\frac{f(e)}{2}$. Substituting this into (1.8) we find after reduction that

$$
\alpha m(x)(\chi(y) m(\sigma(y))-M(y))=\beta M(x)(\chi(y) M(\sigma(y))-m(y)) \text {. }
$$

If we replace $y$ by $\sigma(y)$ in (3.8) and after we multiply equation obtained by $\chi(y)$ and using $\chi(y \sigma(y))=1$ we find

$$
\alpha m(x)(m(y)-\chi(y) M(\sigma(y)))=\beta M(x)(M(y))-\chi(y) m(\sigma(y)))
$$

Subtracting (3.8) from (3.9) we get after some simplifications that 


$$
\begin{aligned}
& (\alpha m(x)+\beta M(x))(\chi(y) m(\sigma(y))-M(y)) \\
= & (\alpha m(x)+\beta M(x))(\chi(y) M(\sigma(y))-m(y)) .
\end{aligned}
$$

Putting $x=e$ in (3.10) we find that $\chi(y) m(\sigma(y))-M(y)$ $=\chi(y) M(\sigma(y))-m(y)$, because $\alpha+\beta=2 c \neq 0$. If $\chi M \circ \sigma-m \neq 0$, then from (3.8) we get $\alpha m(x)=\beta M(x)$ for all $x \in G$. So, for $x=e$ we obtain $\alpha=\beta$ which contradicts the assumption that $f(e) \neq 0$. Thus, $M=\chi m \circ \sigma$ and $m=\chi M \circ \sigma$ from which we see that $g=\frac{m+\chi m \circ \sigma}{2}$ and $f=\left(c+\frac{f(e)}{2}\right) m-\left(c-\frac{f(e)}{2}\right) \chi m \circ \sigma$. We conclude that we deal with case (iii).

(3) $g=m$ and $f_{e}=m a$, where $m$ is multiplicative of $G$ and $a$ is an additive map. From $f_{e}=f-f(e) g$ we get $f=m a+f(e) m=(a+f(e)) m$. Substituting this into (1.8) we find after reduction that

$$
\begin{gathered}
m(x)(a(y) m(y)+\chi(y) a(\sigma(y)) m(\sigma(y))) \\
+m(x)(a(x)+f(e))(\chi(y) m(\sigma(y))-m(y))=0 .
\end{gathered}
$$

If we replace $y$ by $\sigma(y)$ in (3.11) and after we multiply equation obtained by $\chi(y)$ and using $\chi(y \sigma(y))=1$ we find

$$
\begin{gathered}
m(x)(\chi(y) a(\sigma(y)) m(\sigma(y))+a(y) m(y)) \\
+m(x)(a(x)+f(e))(m(y)-\chi(y) m(\sigma(y)))=0 .
\end{gathered}
$$

Subtracting (3.11) from (3.12) we get after some simplifications that

$$
2 m(x)(a(x)+f(e))(\chi(y) m(\sigma(y))-m(y))=0
$$

for all $x, y \in G$. Putting $x=e$ in (3.13) we get $m=\chi m \circ \sigma$, because $2 m(e)(a(e)+f(e))=2.1 .(0+f(e))=2 f(e) \neq 0$. This means that $g=$ $\frac{m+\chi m \circ \sigma}{2}$. Substituting $m=\chi m \circ \sigma$ into (3.11) we deduce that $m(a \circ \sigma+a)=$ 0 . We see that we deal with case (iv) and this completes the proof.

The formulas of Theorem 3.1 implies the following corollary.

Corollary 3.3. Let $G$ be a group, $\sigma: G \longrightarrow G$ a multiplicative function such that $\sigma \circ \sigma=I$, where $I$ denotes the identity map, and $\chi: G \longrightarrow \mathbf{C}$ be a multiplicative function of $G$ such that $\chi(x \sigma(x))=1$ for all $x \in G$. If $f ; g$ : $G \longrightarrow \mathbf{C}$ is a solution of variant of Wilson's functional equation (1.8) such 
that $f \neq 0$, then $g$ is a solution of the variant of d'Alembert's functional equation (3.6).

In the rest of this section we examine the Hyers-Ulam stability of the functional equation (1.8). We shall first recall two variants of Székelyhidi results because it will be useful in the treatment of stability of other functional equations like sine addition formula. The proof of Theorem 3.3 and Theorem 3.4 goes along the same lines as the one in [33] and [34].

Theorem 3.4 (33). Let $V$ be a vector space of $\mathbf{C}$-valued functions on a semigroup $G$, let $V$ be left invariant and suppose that $f$ and $m$ are $\mathbf{C}$-valued functions on $G$. If the function $y \longmapsto f(x y)-f(y) m(x)$ belongs to $V$ for all $x \in G$. Then either $f$ is in $V$ or $m$ is an exponential.

Theorem 3.5 (35). Let $V$ be a vector space of $\mathbf{C}$-valued functions on a semigroup $G$, let $V$ be invariant and suppose that $f$ and $g$ are $\mathbf{C}$-valued functions on $G$ which are linearly independent modulo $V$.

If the function $x \longmapsto f(y x)-f(x) g(y)-f(y) g(x)$ belongs to $V$ for all $y \in G$, then $f(x y)=f(x) g(y)+f(y) g(x)$ for all $x, y \in G$.

In the following theorem we obtain the Hyers-Ulam stability of the functional equation (1.8). The following lemmas will be helpful in the sequel.

Lemma 3.6. Let $\delta \geq 0$, let $G$ be a semigroup with identity element, $\sigma$ : $G \longrightarrow G$ be an homomorphism such that $\sigma \circ \sigma=I$, and $\chi: G \longrightarrow \mathbf{C}$ be a bounded multiplicative function such that $\chi(x \sigma(x))=1$ for all $x \in G$. Suppose that the pair $f, g: G \rightarrow \mathbf{C}$ satisfies

$$
|f(x y)+\chi(y) f(\sigma(y) x)-2 f(x) g(y)| \leq \delta, \text { for all } x, y \in G .
$$

Under these assumptions the following statement hold:

$$
\left|f_{a}(x y)-f_{a}(x) g(y)-f_{a}(y) g(x)\right| \leq|g(x)| \delta+\frac{3}{2} \delta, \text { for all } x, y \in G,
$$

where $f_{a}$ is the function defined in (3.4). 
Proof. For $x, y \in G$ we put $F(x, y)=f(x y)+\chi(y) f(\sigma(y) x)-2 f(x) g(y)$. By using similar computations used in the proof of Theorem 3.1 we get

$$
\begin{gathered}
f(x y z)-f(x) g(y z)+2 f(x) g(y) g(z)-f(x y) g(z)-g(y) f(x z) \\
=-g(y) F(x, z)+\frac{F(x, y z)}{2}+\frac{F(x y, z)}{2}-\frac{F(\sigma(z) x, y)}{2}
\end{gathered}
$$

for all $x, y, z \in G$. From inequality (3.14) and the definition of $f_{a}$ we get the desired result.

The second main result of this section is the next one.

Theorem 3.7. Let $G$ be a semigroup with identity element, $\sigma: G \longrightarrow G$ an involutive homomorphism of $G$ and $\chi: G \longrightarrow \mathbf{C}$ be a unitary character of $G$ such that $\chi(x \sigma(x))=1$ for all $x \in G$. Let the pair $f, g: G \rightarrow \mathbf{C}$ be given. Suppose that the function

$$
(x, y) \longrightarrow f(x y)+\chi(y) f(\sigma(y) x)-2 f(x) g(y)
$$

is bounded. Under these assumptions the following statements hold:

(i) $f=0$ and $g$ arbitrary.

(ii) $f \neq 0$ is bounded and $g$ is bounded.

(iii) $f$ is unbounded, $g$ is bounded and $G$ is an amenable group, then $g \neq 0$ is multiplicative, $g=\chi g \circ \sigma$ and there exists an additive map $a: G \longrightarrow \mathbf{C}$ such that $f-a g$ is bounded and $(a g)(x y)+\chi(y)(a g)(\sigma(y) x)=2(a g)(x) g(y)$ for all $x, y \in G$.

(iv) $f$ is unbounded, $g$ is unbounded. In this case there are the following three possibilities:

(1) $g$ is multiplicative, $g=\chi g \circ \sigma, f=f(e) g$. Furthermore, $f, g$ satisfy equation (1.8).

(2) $g$ is multiplicative, $g=\chi g \circ \sigma, f=a g$, where $a$ is an additive map such that $a \circ \sigma=-a$. Furthermore, $f, g$ satisfy equation (1.8).

(3) $g=\frac{m+\chi m \circ \sigma}{2}$ and $f=\left(c+\frac{f(e)}{2}\right) m-\left(c-\frac{f(e)}{2}\right) m \circ \sigma$, where $m$ is multiplicative.

Proof. If $f=0$ we deal with case (i). So during the rest of the proof we will assume that $f \neq 0$. If $f$ is bounded then by using (3.17) we get $g$ bounded. This is case (ii)

(iii) If $f$ is unbounded and $g$ bounded. We notice here that $g \neq 0$, because if $g=0$ then from (3.17) with $y=e$ we get $f$ bounded, which contradict 
our assumption that $f$ is unbounded. We put $h=f-f(e)$, so $h(e)=0$ and the function

$$
(x, y) \longrightarrow h(x y)-h(x) g(y)-h(y) g(x)
$$

is bounded. Thus, the function $y \longmapsto h(x y)-h(y) g(x)$ is bounded for all $x \in G$. So, by using Theorem 3.4 we get $g=m$ multiplicative and the function defined in (3.18) remains bounded when the right side is multilied by $m\left((x y)^{-1}\right)=m\left(x^{-1}\right) m\left(y^{-1}\right)$, so that the function $(x, y) \longrightarrow$ $\frac{h}{m}(x y)-\frac{h}{m}(x)-\frac{h}{m}(y)$ is bounded. Since $G$ is amenable then from [36] we have $\frac{h}{m}(x)=a(x)+b(x)$ for all $x \in G$, where $a: G \longrightarrow \mathbf{C}$ is an unbounded additive map and $b: G \longrightarrow \mathbf{C}$ is bounded. On the other hand by substituting this into $B(x, y)=h(x y)+\chi(y) h(\sigma(y) x)-2 h(x) g(y)$ we get $a(x y) m(x y)+b(x y) m(x y)+\chi(y)[a(\sigma(y) x) m(\sigma(y) x)+b(\sigma(y) x) m(\sigma(y) x)]=$ $2[a(x) m(x)+b(x) m(x)] m(y)+B(x, y)$ and we find after reduction that the function

$$
\begin{gathered}
\mid a(x) m(x)(\chi(y) m(\sigma(y))-m(y))+a(y) m(x) m(y) \\
+\chi(y) a(\sigma(y)) m(x) m(\sigma(y)) \mid \leq \delta
\end{gathered}
$$

for all $x, y \in G$ and for some $\delta \geq 0$. By replacing $y$ by $\sigma(y)$ in $((3.19))$ and using $\chi(y \sigma(y))=1$ we get

$$
\begin{gathered}
\mid a(x) m(x)(m(y)-\chi(y) m(\sigma(y)))+\chi(y) a(\sigma(y)) m(x) m(\sigma(y)) \\
+a(y) m(x) m(y) \mid \leq \delta .
\end{gathered}
$$

Subtracting (3.19) from (3.20) we get after some simplifications that

$$
|2 a(x) m(x)||(m(y)-\chi(y) m(\sigma(y)))| \leq 2 \delta
$$

for all $x, y \in G$. Since $|m(x)|=1$ and $a$ is unbounded then we get $m(y)=$ $\chi(y) m(\sigma(y)))$ for all $y \in G$.

Now, we will show that $l=a g$ satisfies $l(x y)+\chi(y) l(\sigma(y) x)=2 l(x) m(y)$. For all $x, y \in G$ we have

$$
\begin{aligned}
l(x y)+\chi(y) l(\sigma(y) x)-2 l(x) m(y)= & (a(x)+a(y)) m(x) m(y)+\chi(y)(a(\sigma(y)) \\
& +a(x)) m(\sigma(y)) m(x)-2 a(x) m(x) m(y) \\
= & (a(y)+a(\sigma(y))) m(x) m(y) .
\end{aligned}
$$


Since

$$
|h(x y)+\chi(y) h(\sigma(y))-2 h(x) g(y)| \leq \beta
$$

for some $\beta \geq, h(e)=0, h=m(a+b), m$ is multiplicative, $|m(x)|=1$, $m=\chi m \circ \sigma$ and $b$ is bounded then if we put $x=e$ in (3.22) we get

$$
|m(y) a(y)+m(y) b(y)+\chi(y) m(\sigma(y)) a(\sigma(y))+\chi(y) m(\sigma(y)) b(\sigma(y))| \leq \beta .
$$

This means that the function $y \longrightarrow|a(y)+a(\sigma(y))|$ is bounded. Since $a+a \circ \sigma$ is an additive map then we get $a(y)+a(\sigma(y)=0$ for all $y \in G$ and we conclude that $l(x y)+\chi(y) l(\sigma(y) x)=2 l(x) g(y)$ for all $x, y \in G$, and we see that we deal with case (iii).

If $f, g$ are unbounded, then by using (3.15) we get that either $f_{a}=0$ for all $a \in G$ or $f_{a}$ is unbounded for all $a \in G$. Indeed, if there exists $a \in G$ with $f_{a} \neq 0$ and $f_{a}$ bounded, so from inequality (3.15) with $x=x_{0}$ where $f_{a}\left(x_{0}\right) \neq 0$ we get $g$ bounded which contradicts the assumption that $g$ is unbounded.

In this case we have the following possibilities:

If $f_{a}=0$ for all $a \in G$ then $f(x y)=f(x) g(y)$ for all $x, y \in G$ and this implies that $g$ is multiplicative and $f=f(e) g$. Substituting this into (3.17) we get after reduction that $|g(x)| \chi(y) g(\sigma(y))-g(y) \mid \leq \gamma$ for all $x, y \in G$ and for some $\gamma \geq 0$. Since $g$ is unbounded we deduce that $g=\chi g \circ \sigma$. So, $g$ satisfies equation (1.9), and the pair $f, g$ satisfies equation (1.8). We deal with case (iv)(1).

If there exists $a \in G$ such that $f_{a} \neq 0$, then by using the above notice we get $f_{a}$ is unbounded for all $a \in G$. For the rest of the proof we put $a=e$ and we will discuss two cases.

First Case: If $f_{e}, g$ are linearly dependent modulo the spaces of complex bounded function on $G$ (see [35]), then there exists a constant $\lambda \in \mathbf{C}^{*}$ and a bounded function $b$ on $G$ such that $g=\frac{1}{2 \lambda} f_{e}+b$. Substituting this into inequality (3.15) we get

$\left.\left|f_{e}(x y)-f_{e}(x)\left[\frac{1}{2 \lambda} f_{e}(y)+b(y)\right]-f_{e}(y)\left[\frac{1}{2 \lambda} f_{e}(x)+b(x)\right]\right| \leq \mid \frac{1}{2 \lambda} f_{e}(x)+b(x)\right] \mid \delta+\frac{3}{2} \delta$ 
for all $\mathrm{x}, \mathrm{y} \in G$, so we have

$$
\left.\left|f_{e}(x y)-\left(\frac{1}{\lambda} f_{e}(x)+b(x)\right) f_{e}(y)\right| \leq\left|f_{e}(x)\right||b(y)|+\mid \frac{1}{2 \lambda} f_{e}(x)+b(x)\right] \mid \delta+\frac{3}{2} \delta .
$$

Thus the function $y \longrightarrow f_{e}(x y)-\left(\frac{1}{\lambda} f_{e}(x)+b(x)\right) f_{e}(y)$ is bounded for all $x \in G$. Since $f_{e}$ is unbounded then from Theorem 3.4 (with $V$ is the space of bounded function on $G$ ) we get $m=\frac{1}{\lambda} f_{e}+b$ multiplicative, $f_{e}=\lambda m-\lambda b$, $g=\frac{m}{2}+\frac{b}{2}$ and $f=f_{e}+f(e) g=\left(\lambda+\frac{f(e)}{2}\right) m+\left(\frac{f(e)}{2}-\lambda\right) b=\alpha m+\beta b$ Substituting this into bounded function $B(x, y)=f(x y)+\chi(y) f(\sigma(y) x)-$ $2 f(x) g(y)$ we find after reduction that

$\alpha m(x)[\chi(y) m(\sigma(y))-b(y)]=\beta b(x) m(y)+\beta b(x) b(y)-\beta \chi(y) b(\sigma(y) x)+B(x, y)$ $(3.23)$

for all $x, y \in G$. Since $b$ is bounded, $m$ is unbounded and $|\chi(y)|=1$ then there exists $a \in G$ such that $\chi(a) m(\sigma(a))-b(a) \neq 0$. From (3.23) we conclude that $m$ is a bounded multiplicative and this case does not apply, because $m$ is unbounded. So we have the second case:

Case 2: $f_{e}, g$ are linearly independent modulo the spaces of complex bounded function on $G$. From inequality (3.15) and Theorem 3.5, (with $V$ is the space of bounded function on $G$ ) reveals that the pair $\left(f_{e}, g\right)$ is a solution of the sine addition formulas

$$
f_{e}(x y)=f_{e}(x) g(y)+f_{e}(y) g(x)
$$

for all $x, y \in G$, so we known from [[40], Corollary 4.4] that there are only the following possibilities:

(1) $f_{e}=\mathrm{cm}$ and $g=\frac{m}{2}$ for some multiplicative function $m: G \longrightarrow \mathbf{C}$. Here $f=f_{e}+f(e) g=\left(c+\frac{f_{e}}{2}\right) m=\gamma m$. Substituting this into bounded function $B(x, y)=f(x y)+\chi(y) f(\sigma(y) x)-2 f(x) g(y)$ we find after reduction that $\beta \chi(y) m(\sigma(y)) m(x)=B(x, y)$. This means that $m$ is a bounded multiplicative and this case does not apply, because $m$ is unbounded.

(2) $g=m$ and $f_{e}=a m$ for some multiplicative function $m: G \longrightarrow \mathbf{C}$ and $a: G \longrightarrow \mathbf{C}$ an additive map. In this case $f=(a+f(e)) m$, so we find after reduction that the bounded function:

$$
f(x y)+\chi(y) f(\sigma(y) x)-2 f(x) g(y)=(a(x)+a(y)+f(e)) m(x) m(y)
$$


Solutions and stability of a variant of Wilson's functional equation 339

$$
\begin{aligned}
& +\chi(y)[(a(\sigma(y))+a(x)+f(e)) m(\sigma(y)) m(x)-2(a(x)+f(e)) m(x) m(y)] \\
& =(a(x)+f(e)) m(x)(\chi(y) m(\sigma(y))-m(y))+m(x)[\chi(y) a(\sigma(y)) m(\sigma(y))+a(y) m(y)] .
\end{aligned}
$$

If we replace $y$ with $\sigma(y)$ in (3.25), and after we multiply equation obtained by $\chi(y)$ and using $\chi(y \sigma(y))=1$ we get

$(a(x)+f(e)) m(x)(m(y)-\chi(y) m(\sigma(y)))+m(x)(a(y) m(y)+\chi(y) a(\sigma(y)) m(\sigma(y))$

which is also a bounded function. Subtracting (3.25) from (3.26) we get after some simplifications that the function $(x, y) \longmapsto m(x)(a(x)+f(e))(m(y)-$ $\chi(y) m(\sigma(y)))$ is bounded. Since $f=m(a+f(e))$ is unbounded then we get $m=\chi m \circ \sigma$. Now, we will verify that the pair $(f, g)$ is a solution of equation (1.8). for all $x, y \in G$ we have

$$
\begin{gathered}
f(x y)+\chi(y) f(\sigma(y) x)-f(x) g(y) \\
=(a(x)+a(y)+f(e)) m(x) m(y) \\
+\chi(y)[a(\sigma(y))+a(x)+f(e)) m(\sigma(y)) m(x)]-2(a(x)+f(e)) m(x) m(y) \\
=(a(y)+a(\sigma(y))) m(x) m(y) .
\end{gathered}
$$

Since $(x, y) \longmapsto f(x y)+\chi(y) f(\sigma(y) x)-f(x) g(y)$ is a bounded function, then we have $(x, y) \longmapsto(a(y)+a(\sigma(y))) m(x) m(y)$ is also bounded. Since $m$ is unbounded then we get the desired result, so we see that we deal with case (iv) (2).

(3) There exit two different characters $m, M$ and a constant $c \in \mathbf{C}^{*}$ such that $g=\frac{m+M}{2}$ and $f_{e}=c(m-M)$. In this case $f=f_{e}+f(e) g=$ $\left(c+\frac{f(e)}{2}\right) m-\left(c-\frac{f(e)}{2}\right) m=\alpha m-\beta M$, where $\alpha=c+\frac{f(e)}{2}$ and $\beta=c-\frac{f(e)}{2}$.

Substituting this into bounded function $B(x, y)=f(x y)+\chi(y) f(\sigma(y) x)-$ $2 f(x) g(y)$ we find after reduction that

$(\operatorname{ax}(x)(\chi(y) m(\sigma(y))-M(y))+\beta M(x)(m(y)-\chi(y) M(\sigma(y)))=B(x, y)$. 
If we replace $y$ with $\sigma(y)$ in (3.27), and after we multiply equation obtained by $\chi(y)$ and using $\chi(y \sigma(y))=1$ we get

$\alpha m(x)(m(y))-\chi(y) M(\sigma(y)))+\beta M(x)(\chi(y) m(\sigma(y))-M(y))=\chi(y) B(x, \sigma(y))$.

If we add (3.27) to (3.28) we get after some simplifications that the function $(x, y) \longmapsto(\alpha m(x)+\beta M(x))[(m(y)-\chi(y) M(\sigma(y)))+(\chi(y) m(\sigma(y))-$ $M(y))]$ is bounded. Since $\alpha m+\beta M=2 c g+\frac{f(e)}{c} f_{e}$ and $g, f_{e}$ are linearly independent modulo the space of complex bounded functions on $G$, $\alpha m+\beta M=2 c g+\frac{f(e)}{c} f_{e}$ is unbounded then we get $m-\chi M \circ \sigma=M-\chi m \circ \sigma$. Now, the bounded function (3.27) can be written as follows

$f(x y)+\chi(y) f(\sigma(y) x)-2 f(x) g(y)=(\alpha m(x)-\beta M(x))(\chi(y) m(\sigma(y))-M(y))$

$$
=f(x)(\chi(y) m(\sigma(y))-M(y))
$$

Since $f$ is assumed to be unbounded then we get $\chi(y) m(\sigma(y))=M(y)$ for all $y \in G$ and $g$ take the expression: $g=\frac{m+\chi m \circ \sigma}{2}$. Equation (3.29) show that the pair $(f, g)$ satisfies equation (1.8). We see that we deal with case (iv) (3) and this completes the proof. $\square$ As an application we get the superstability of the functional equation (3.6).

Corollary 3.8. Let $\delta \geq 0$. Let $G$ be a semigroup with identity element, $\sigma: G \longrightarrow G$ an involutive homomorphism and $\chi: G \longrightarrow \mathbf{C}$ be a unitary character of $G$ such that $\chi(x \sigma(x))=1$ for all $x \in G$. Let $f: G \rightarrow \mathbf{C}$ such that

$$
|f(x y)+\chi(y) f(\sigma(y) x)-2 f(x) f(y)| \leq \delta
$$

for all $x, y \in G$. Then either $f$ is bounded or $f$ satisfies equation (3.6)

In [23], the authors presented some rich ideas on the study of the superstability of symmetrized multiplicative Cauchy equation

$$
f(x y)+f(y x)=2 f(x) f(y) x, y \in G .
$$

However, we have formulate the problem as an open problem. The solutions of equation (3.31) are multiplicative functions (see for exapmle [30]. In the following, we give the affirmative answer. If we put $\chi=1$ and $\sigma=I$ in Corollary 3.8, where $I$ denotes the identity map we get 
Corollary 3.9. Let $\delta \geq 0$. Let $G$ be a group with identity element. Let $f: G \rightarrow \mathbf{C}$ such that

$$
|f(x y)+f(y x)-2 f(x) f(y)| \leq \delta
$$

for all $x, y \in G$. Then either $f$ is bounded or $f$ is multiplicative.

\section{Acknowledgements.}

The authors thank very much the referees for their helpful comments. We would also like to thank the professor H. Stetkær. His help for the final version of this paper is greatly appreciated.

\section{References}

[1] J. Aczél and J. Dhombres, Functional equations in several variables. With applications to mathematics, information theory and to the natural and social sciences. Encyclopedia of Mathematics and its Applications, 31. Cambridge University Press, Cambridge, (1989).

[2] R. Badora, Stability Properties of Some Functional Equations. In: Themistocles Rassias, Janusz Brzdek (ed.) Functional Equations in Mathematical Analysis, pp.3-13. Springer Optimization and Its Applications, 52, (2011).

[3] R. Badora, On the stability of a functional equation for generalized trigonometric functions. In: Th. M. Rassias (ed.) Functional Equations and Inequalities, pp.1-5. Kluwer Academic Publishers, (2000).

[4] J. A. Baker, The stability of the cosine equation, Proc. Amer. Math. Soc., 80, pp. 411-416, (1980).

[5] B. Bouikhalene and E. Elqorachi, Stability of the spherical functions, Georgian Math. J., — DOI: https://doi.org/10.1515/gmj-2015-0052, 23 (2016).

[6] B. Bouikhalene, E. Elqorachi and J. M. Rassias, The superstability of d'Alembert's functional equation on the Heisenberg group, App. Math. Letters, 23, No.1, pp. 105-109, (2010).

[7] T.M.K. Davison, D'Alembert's functional equation on topological groups. Aequationes Math., 76, pp. 33-53. (2008). 
[8] T.M.K. Davison, D'Alembert's functional equation on topological monoids. Publ. Math. Debrecen, 75, 1/2, pp. 41-66, (2009).

[9] Y. Dilian, Factorization of cosine functions on compact connected groups. Math. Z., 254, No. 4, pp. 655-674, (2006).

[10] Y. Dilian, Functional equations and Fourier analysis, Canadian Mathematical Bulletin, 56, pp. 218, (2011).

[11] Y. Dilian, Cosine functions revisited. Banach J. Math. Anal., 5, pp. 126-130, (2011).

[12] R. Ebanks, Bruce and H. Stetkær, On Wilson's functional equations, Aequationes Math., 89, pp. 339-354, (2015).

[13] E. Elqorachi and M. Akkouchi, The superstability of the generalized dAlembert functional equation, Georgian Math. J., 10, pp. 503-508, (2003).

[14] G. L. Forti, Hyers-Ulam stability of functional equations in several variables, Aequationes Math., 50, pp. 143-190, (1995).

[15] F. Peter de Place, d'Alembert's and Wilson's functional equations on Lie groups, Aequationes Math., 67, pp. 12-25, (2004).

[16] R. Ger, Superstability is not natural, Rocznik Nauk.-Dydakt. Prace Mat., 159, No. 13, pp. 109-123, (1993).

[17] R. Ger and P. Šemrl, The stability of the exponential equation, Proc. Amer. Soc., 124, pp. 779-787, (1996).

[18] D. H. Hyers, G. I. Isac and Th. M. Rassias, Stability of Functional Equations in Several Variables, Birkhäuser, Basel,, (1998).

[19] S.-M. Jung, Hyers-Ulam-Rassias Stability of Functional Equations in Mathematical Analysis, Hadronic Press, Inc., Palm Harbor, Florida,, (2003).

[20] PL. Kannappan, The functional equation $f(x y)+f\left(x y^{-1}\right)=2 f(x) f(y)$ for groups, Proc. Amer. Math. Soc., 19, pp. 69-74, (1968).

[21] G. H. Kim, On the stability of trigonometric functional equations, Advances in Difference Equations, Vol. (2007), Article ID 90405, 10 pages. 
[22] G. H. Kim, On the stability of the Pexiderized trigonometric functional equation, Applied Mathematics and Computation, 203, No. 1, pp. 99105, (2008).

[23] A. Redouani, E. Elqorachi and M. Th. Rassias, The superstability of d'Alembert's functional equation on step 2-nilpotent groups, Aequationes math., 74, No. 3, pp. 226-241, (2007).

[24] H. Stetkær, A link between Wilson's and d'Alembert's functional equations, Aequationes Math., 90, pp. 407-409, (2016).

[25] H. Stetkær, A variant of d'Alemberts functional equation, Aequationes Math., 89, pp. 657-662, (2015).

[26] H. Stetkær, D'Alembert's functional equation on groups, Banach Center Publ., 99, pp. 173-191, (2013).

[27] H. Stetkær, Functional equations on groups, World Scientific, New Jersey, London, Singapore, Beijing, Shanghai, Hong Kong, Taipei, Chennai, (2013).

[28] H. Stetkær, Properties of d'Alembert functions, Aequationes Math., 77, pp. 281-301, (2009).

[29] H. Stetkær, d'Alembert's and Wilson's functional equations on step 2 nilpotent groups, Aequationes Math., 67, No. 3, pp. 241-261, (2004).

[30] H. Stetkær, On multiplicative maps, Semigroup Forum, 63, pp. 466468, (2001).

[31] H. Stetkær, On a variant of Wilson's functional equation on groups, Aequationes Math., 68, No. 3, pp. 160-176, (2004).

[32] L. Székelyhidi, On a stability theorem, C. R. Math. Acad. Sc. Canada, 3, pp. 253-255, (1981).

[33] L. Székelyhidi, On a theorem of Baker, Lawrence and Zorzitto, Proc. Amer. Math. Soc., 84, pp. 95-96, (1982).

[34] L. Székelyhidi, The stability of dAlembert-type functional equations, Acta Sci. Math. Szeged 44, pp. 313-320, (1982).

[35] L. Székelyhidi, The stability of the sine and cosine functional equations, Proc. Amer. Math. Soc., 110, pp. 109-115, (1990). 
[36] L. Székelyhidi, Fréchet equation and Hyers's theorem on noncommutative semigroups, Ann. Polon. Math., 48, pp. 183-189, (1988).

\title{
Elhoucien Elqorachi
}

Department of Mathematics

Faculty of Sciences

Ibn Zohr University

Agadir,

Morocco

e-mail : e.elqorachi@uiz.ac.ma

and

\author{
Ahmed Redouani \\ Department of Mathematics \\ Faculty of Sciences \\ Ibn Zohr University \\ Agadir, Morocco \\ e-mail : Redouani_ahmed@yahoo.fr
}

